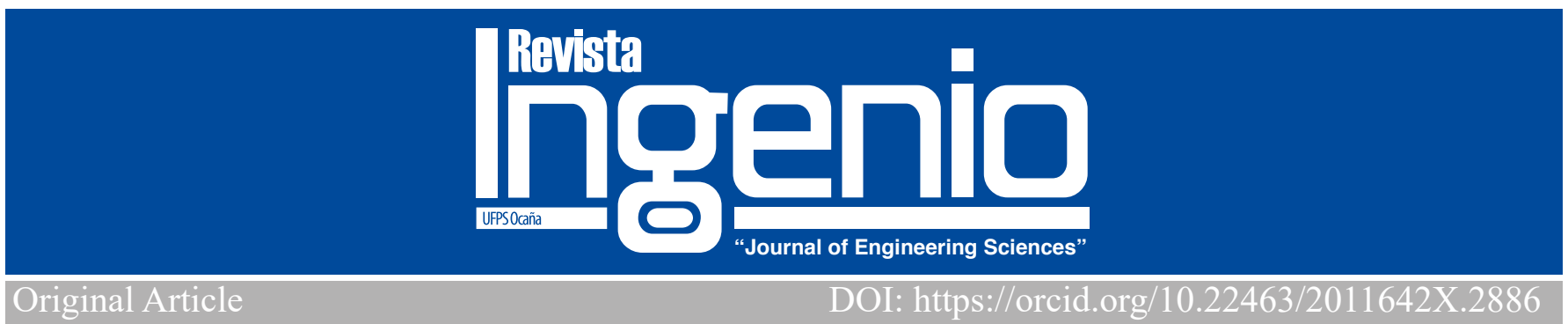

\title{
Diseño y análisis experimental para determinar la porosidad de materiales adsorbentes compuestos
}

Experimental design and analysis to determine the porosity of composite adsorbent materials

\section{MSc. Eduar Pérez Rojas ${ }^{1}$}

${ }^{1}$ Universidad del Norte, Colombia, Orcid: https://orcid.org/0000-0003-2971-3453,Email: eeperez@uninorte.edu.co

Como citar: E. Pérez-Rojas, "Diseño y análisis experimental para determinar la porosidad de materiales adsorbents compuestos", Revista Ingenio, vol. 15 (1), pp.23-30, 2018

\begin{tabular}{ll}
\hline RESUMEN \\
\hline Palabras claves: & $\begin{array}{l}\text { Los sistemas de refrigeración por adsorción que usan como fuente calor residual, dependen de la calidad del lecho adsorbente donde } \\
\text { se utilizan sales anhidras cuya conductividad térmica y porosidad son bajas; por esta razón se impregnan con materiales como el } \\
\text { grafito o el carbón activado. En este trabajo se desarrolló un diseño de experimentos de tipo diseño central compuesto (CCD, por sus }\end{array}$ \\
Adsorción, carbón & siglas en inglés, inicialmente se implementó un diseño factorial completo $2 \mathrm{k}$ que permitió determinar cuan significativos eran cada \\
activado, cloruro de calcio, & uno de los factores y su influencia sobre la variable de respuesta. Este permitió conocer que los factores tiempo, fracción de material \\
diseño central compuesto, & y tipo de material, son los más influyentes con un valor p menor a 0.05. posteriormente mediante el diseño CCD, se estableció una \\
diseño factorial $2^{\mathrm{k}}$, grafito & superficie de respuesta y una región óptima donde el material tiene una porosidad alta con un mínimo costo de producción, esto tiene \\
expandido, porosidad. & gran implicación en la simplificación del desarrollo de procedimientos para producir materiales adsorbentes compuestos de mayor \\
& eficiencia. Asimismo, de determinó que los compuestos con un porcentaje mayor a 50\% en la fracción de material de impregnación \\
& mejoran las condiciones de la porosidad.
\end{tabular}

\section{ABSTRACT}

Key words:

Adsorption, activated carbon, calcium chloride, composite core design, $2^{\mathrm{k}}$ factorial design, expanded graphite, porosity.

\begin{abstract}
Adsorption cooling systems that use residual heat as a source depend on the quality of the adsorbent bed where anhydrous salts are used whose thermal conductivity and porosity are low; for this reason, they are impregnated with materials such as graphite or activated carbon. In this work, a design of experiments of the central compound design type (CCD) was developed, initially a $2 \mathrm{k}$ full factorial design was implemented that allowed determining how significant each of the factors were and their influence on the response variable. Time factors, material fraction and type of material are the most influential with a p value less than 0.05 . Later, through the CCD design, a response surface and an optimal region were established where the material has a high porosity with a minimum cost. of production, this has great implication in the simplification of the development of procedures to produce adsorbent materials composed of higher efficiency. Likewise, it was determined that the compounds with a percentage greater than $50 \%$ in the fraction of impregnation material improve the conditions of porosity.
\end{abstract}

\section{Introducción}

En la actualidad los sistemas de refrigeración son utilizados en múltiples procesos que van desde la conservación de alimentos en su transporte y comercialización, hasta en la creación de ambientes agradables para el confort humano. Muchos de esos sistemas de refrigeración operan bajo ciclos de compresión a vapor que usan como fuente de energía principal la electricidad, aunque esta tecnología ha tenido grandes desarrollos en los últimos tiempos [1]. Es por esta razón que en los últimos 40 años se han retomado estudios de sistemas que promuevan efecto frigorífico y sean más amigables con el ambiente como los sistemas de refrigeración por sorción [2-3-4-5-6]. Buscando mejorar la conductividad térmica y la porosidad de los materiales adsorbentes se propuso impregnaciones de dichos materiales con grafito expandido, en proporciones de masa que no superen el $23 \%$; J.K. Kiplagat propone impregnar cloruro de litio con grafito expandido con el cual se registró la influencia sobre la capacidad de enfriamiento por unidad de masa de sal en un $20 \%$ [7].

Oliveira et al. [8] Estudió un prototipo a escala de laboratorio con un compuesto adsorbente $\mathrm{NaBr}$ grafito expandido obteniendo $219 \mathrm{~kJ} / \mathrm{kg}$, de potencia de refrigeración a $5^{\circ} \mathrm{C}$ y $510 \mathrm{k} . / \mathrm{kg}$ de refrigeración a $15^{\circ} \mathrm{C}$ con una fuente de calor a $65{ }^{\circ} \mathrm{C}$ y temperatura de condensación a $30^{\circ} \mathrm{C}$. Con las mismas condiciones de fuente de calor y evaporador el sistema consiguió una potencia de refrigeración entre 75 y 
$79 \mathrm{Kw} / \mathrm{m} 3$, con un coeficiente de operación (COP) que va desde 0.43 a 0.46 cuando la temperatura de enfriamiento se encontraba a $15{ }^{\circ} \mathrm{C}$, lo que hace que el grafito expandido sea un buen complemento para aumentar la conductividad térmica y la porosidad de los materiales adsorbentes como lo demostró. El diseño de experimentos (DOE) ha sido una de las principales técnicas utilizadas para analizar y determinar factores significativos del proceso que influyen en el rendimiento del proceso [9]. Los principales propósitos de su implementación incluyen la determinación de factores que son más impresionantes en los resultados, la determinación de factores influyentes en los factores del proceso para lograr la mejor respuesta y la reducción de la variabilidad. Para realizar el análisis experimental se propone evaluar los factores independientes que inciden en la preparación de la impregnación del material adsorbente con el material que mejora la conductividad térmica y la porosidad del mismo, (tiempo de mezclado, cantidad de material de impregnación, tipo de material de impregnación y fuerza de compactación del bloque de material adsorbente;) en la variable de respuesta (porosidad), el comportamiento de esta variable se analizó con diseño factorial $2 \mathrm{k}$, para realizar un cribado, para luego extenderlo a un diseño compuesto central con el fin de identificar los coeficientes de una ecuación polinómica que relacione la variable de respuesta con los factores.

\section{Materiales y métodos}

\subsection{Preparación de la muestra}

La muestra se preparó como se muestra en la figura 1; impregnando cloruro de calcio con carbón activado y grafito expandido, el proceso inicio con la expiación del grafito que se llevó a $800{ }^{\circ} \mathrm{C}$ durante dos minutos de acuerdo con el procedimiento dado por Oliveria et al. [10].

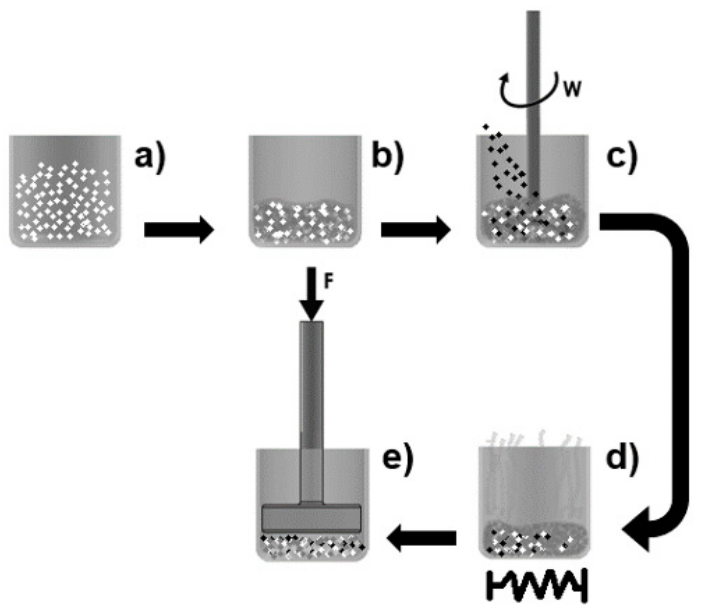

Figura 1. Esquema de la preparación de la muestra a) masa de CaCl_2, b) hidratación, c) impregnación con CA o GE, d) deshidratación, e) compactación.
Posteriormente se realizó la homogenización de las proporciones de cloruro de calcio impregnadas con carbón activado y grafito expandido con agua destilada en un Agitador de laboratorio mecánico de hélice analógico para líquido viscoso a $400 \mathrm{rpm}$, y niveles de factor de tiempo mínimo $5 \mathrm{~min}$ y máximo $15 \mathrm{~min}$, los niveles del factor se muestren en la tabla 1, luego las muestras se llevaron al horno mufla para evaporar el agua en la mezcla a $120^{\circ} \mathrm{C}$ durante 24 horas según el procedimiento propuesto por J.K. Kiplagat et al [11]. Una vez las muestras se encontraban libres de humedad se compactaron con una fuerza de 100 y $300 \mathrm{New}$, para los niveles bajo y alto del factor fuerza.

\subsection{Configuración experimental.}

Los rangos de operación de los factores variaron dependiendo de las restricciones y característica de cada uno de ellos; ya que en la literatura no se especifica los tiempos de homogenización para la impregnación del material adsorbente se decidió mantener fija la velocidad angular en 400 revoluciones por minuto y variar el tiempo de operación de la mezcladora de 5 a 15 minutos.

La masa de cloruro de calcio se mantuvo fija en 70 gramos mientras que la masa de impregnación de los materiales que mejoran la conductividad térmica y la porosidad, variaron entre 5 y 10 gramos; para eliminar la mayor cantidad de aire y romper los grumos formados en el material adsorbente mejorado se aplicaron cargas entre 100 y 300 Newton; los factores del diseño experimental se muestran en la tabla 1.

Tabla 1. Factores experimentales

\begin{tabular}{|c|c|c|c|c|c|}
\hline \multicolumn{6}{|c|}{ Niveles de los factores } \\
\hline Codificación & Factor & Unidades & $\begin{array}{l}\text { Niveles } \\
\text { Bajo }(-1,0)\end{array}$ & Alto $(1,0)$ & Tipo \\
\hline A & Tiempo & $\min$ & 5 & 15 & Cuantitativo \\
\hline B & Fracción & $\mathrm{gr}$ & 5 & 10 & Cuantitativo \\
\hline $\mathrm{C}$ & Fuerza & New & 100 & 300 & Cuantitativo \\
\hline $\mathrm{D}$ & Material & - & $\mathrm{CA}$ & $\mathrm{GE}$ & Cualitativo \\
\hline
\end{tabular}

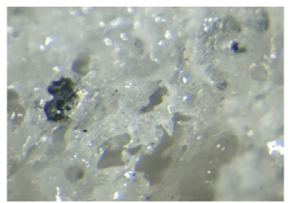

b)

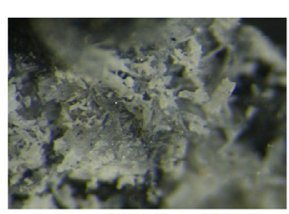

Figura 2. Cloruro de calcio impregnado, aumento 67.2X, a) Carbón activado, b) Grafito expandido.

Para determinar la porosidad del material adsorbente ecuación 1 descrito por Han and Lee, [12] donde se midió el volumen final del material compuesto $\left(\mathrm{V}_{\mathrm{B}}\right)$ con el fin de determinar la densidad aparente del bloque $\left(\rho_{B}\right)$, densidad aparente del grafito expandido $\left(\rho_{(E G} \dot{o} A C\right)$ y la fracción de masa del material de impregnación $\left(f_{(E G \quad O A C}\right)$ cuyos valores se determinaron según las ecuaciones $2,3,4$, que son coeficientes de la ecuación 1 , como resultado se obtuvieron muestras impregnadas como se observa en la figura 2. 


$$
\begin{aligned}
& \emptyset=1-\frac{\rho_{E G \text { ó } C A}}{\rho_{g \text { ó } c a}}-\frac{\left(1-f_{E G \text { ó } C}\right) \rho_{E G \text { ó } C A}}{f_{E G \text { ó } A C}} \frac{\bar{v}_{S}}{M W_{S}} \\
& \rho_{B}=\frac{m_{E G \text { ó } A C}+m_{S}}{V_{B}} \\
& \rho_{E G \text { o } C A}=\frac{m_{E G \text { ó } A C}}{V_{B}} \\
& f_{E G \dot{O} C A}=\frac{m_{E G \delta A C}}{m_{E G O A C}+m_{S}}
\end{aligned}
$$

(1) y tres de carácter cuantitativo, las variables continúas controladas fueron: tiempo de mezclado (Tiempo), cantidad de material imprégnate (Fracción) y fuerza de compactación

\subsection{Condiciones experimentales.}

Durante el proceso de preparación de la muestra se controlaron cuatro variables; una de carácter cualitativo
(Fuerza); el factor cualitativo fue el tipo de imprégnate (Tipo). Los experimentos fueron realizados de acuerdo con un diseño factorial $2 \mathrm{k}$ como se muestra en la tabla 2 , con el cual se determinó la significancia de los factores. Luego se desarrolló un diseño compuesto central CCD, que utilizó las condiciones experimentales de un diseño factorial $2 \mathrm{k}$, más las condiciones experimentales centrales y los puntos estrella (alfa) que muestran en la tabla 6, para observar la curvatura del modelo y determinar el modelo de regresión utilizando el software Statgraphics ${ }^{\circledR}$.

Tabla 2. Valores medios de las variables independientes y sus niveles codificados.

\begin{tabular}{lllll}
\hline Condición experimental & Tiempo (min) & Fracción $(\mathrm{gr})$ & Fuerza $(\mathrm{New})$ & Material \\
\hline 1 & $15(1,0)$ & $10(1,0)$ & $100(-1,0)$ & $\mathrm{CA}(-1,0)$ \\
2 & $15(1,0)$ & $5(-1,0)$ & $300(1,0)$ & $\mathrm{CA}(-1,0)$ \\
3 & $15(1,0)$ & $5(-1,0)$ & $300(1,0)$ & $\mathrm{GE}(1,0)$ \\
4 & $5(-1,0)$ & $10(1,0)$ & $300(1,0)$ & $\mathrm{GE}(1,0)$ \\
5 & $5(-1,0)$ & $5(-1,0)$ & $100(-1,0)$ & $\mathrm{CA}(-1,0)$ \\
6 & $15(1,0)$ & $5(-1,0)$ & $100(-1,0)$ & $\mathrm{GE}(1,0)$ \\
7 & $15(1,0)$ & $5(-1,0)$ & $100(-1,0)$ & $\mathrm{CA}(-1,0)$ \\
8 & $5(-1,0)$ & $10(1,0)$ & $100(-1,0)$ & $\mathrm{CA}(-1,0)$ \\
9 & $15(1,0)$ & $10(1,0)$ & $300(1,0)$ & $\mathrm{GE}(1,0)$ \\
10 & $15(1,0)$ & $10(1,0)$ & $100(-1,0)$ & $\mathrm{GE}(1,0)$ \\
11 & $5(-1,0)$ & $10(1,0)$ & $300(1,0)$ & $\mathrm{CA}(-1,0)$ \\
12 & $5(-1,0)$ & $5(-1,0)$ & $100(-1,0)$ & $\mathrm{GE}(1,0)$ \\
13 & $15(1,0)$ & $10(1,0)$ & $300(1,0)$ & $\mathrm{CA}(-1,0)$ \\
14 & $5(-1,0)$ & $5(-1,0)$ & $300(1,0)$ & $\mathrm{CA}(-1,0)$ \\
15 & $5(-1,0)$ & $5(-1,0)$ & $300(1,0)$ & $\mathrm{GE}(1,0)$ \\
16 & $5(-1,0)$ & $10(1,0)$ & $100(-1,0)$ & $\mathrm{GE}(1,0)$ \\
\hline
\end{tabular}

\section{Resultados y discusiones}

\subsection{Diseño factorial $2 \mathrm{k}$.}

Luego de obtener los datos experimentales expuestos en la tabla 3, se desarrolló un análisis de cribado para determinar la significancia de los factores que inciden en la variable respuesta.
Al analizar el grafico de probabilidad normal para los efectos figura 3 , se encontró que los factores significativos fueron: A, B, D, y las interacciones AD, BD. Estos resultados se verificaron con la exclusión de las interacciones no significativas en el análisis de varianza ANOVA como se muestra en las tablas 4 y 5 .

Tabla 3. Resultados experimentales.

\begin{tabular}{llllll}
\hline Condición experimental & Tiempo $(\mathrm{min})$ & Fracción $(\mathrm{gr})$ & Fuerza $(\mathrm{New})$ & Material & Porosidad $(\emptyset)$ \\
\hline 1 & $15(1,0)$ & $10(1,0)$ & $100(-1,0)$ & CA(-1,0) & 1,0004642 \\
2 & $15(1,0)$ & $5(-1,0)$ & $300(1,0)$ & CA(-1,0) & 1,00065853 \\
3 & $15(1,0)$ & $5(-1,0)$ & $300(1,0)$ & GE $(1,0)$ & 1,00075374 \\
4 & $5(-1,0)$ & $10(1,0)$ & $300(1,0)$ & GE $(1,0)$ & 1,00086209 \\
5 & $5(-1,0)$ & $5(-1,0)$ & $100(-1,0)$ & CA(-1,0) & 1,00147187 \\
6 & $15(1,0)$ & $5(-1,0)$ & $100(-1,0)$ & GE $(1,0)$ & 1,00080205 \\
7 & $15(1,0)$ & $5(-1,0)$ & $100(-1,0)$ & CA(-1,0) & 1,00118038 \\
8 & $5(-1,0)$ & $10(1,0)$ & $100(-1,0)$ & CA(-1,0) & 1,00120693 \\
9 & $15(1,0)$ & $10(1,0)$ & $300(1,0)$ & GE $(1,0)$ & 1,00066315 \\
10 & $15(1,0)$ & $10(1,0)$ & $100(-1,0)$ & GE $(1,0)$ & 1,0005486
\end{tabular}




\begin{tabular}{llllll}
11 & $5(-1,0)$ & $10(1,0)$ & $300(1,0)$ & $\mathrm{CA}(-1,0)$ & 1,00097333 \\
12 & $5(-1,0)$ & $5(-1,0)$ & $100(-1,0)$ & $\mathrm{GE}(1,0)$ & 1,00059019 \\
13 & $15(1,0)$ & $10(1,0)$ & $300(1,0)$ & $\mathrm{CA}(-1,0)$ & 1,00045717 \\
14 & $5(-1,0)$ & $5(-1,0)$ & $300(1,0)$ & $\mathrm{CA}(-1,0)$ & 1,00142182 \\
15 & $5(-1,0)$ & $5(-1,0)$ & $300(1,0)$ & $\mathrm{GE}(1,0)$ & 1,00071091 \\
16 & $5(-1,0)$ & $10(1,0)$ & $100(-1,0)$ & $\mathrm{GE}(1,0)$ & 1,00058589 \\
\hline
\end{tabular}

Tabla 4. Análisis de varianza (ANOVA) para los factores.

\begin{tabular}{llllll}
\hline Fuente & Suma de Cuadrados & Gl & Cuadrado Medio & Razón-F & Valor-P \\
\hline A: Tiempo & $3,29247 \mathrm{E}-7$ & 1 & $3,29247 \mathrm{E}-7$ & 18,31 & 0,0027 \\
B: Fracción & $2,08878 \mathrm{E}-7$ & 1 & $2,08878 \mathrm{E}-7$ & 11,61 & 0,0093 \\
C: Fuerza & $7,62893 \mathrm{E}-9$ & 1 & $7,62893 \mathrm{E}-9$ & 0,42 & 0,5331 \\
D: Material & $3,35706 \mathrm{E}-7$ & 1 & $3,35706 \mathrm{E}-7$ & 18,67 & 0,0025 \\
AD & $3,39925 \mathrm{E}-7$ & 1 & $3,39925 \mathrm{E}-7$ & 18,90 & 0,0025 \\
BD & $1,28487 \mathrm{E}-7$ & 1 & $1,28487 \mathrm{E}-7$ & 7,14 & 0,0282 \\
CD & $1,0171 \mathrm{E}-7$ & 1 & $1,0171 \mathrm{E}-7$ & 5,66 & 0,0447 \\
\hline Error total & $1,43879 \mathrm{E}-7$ & 8 & $1,79848 \mathrm{E}-8$ & & \\
\hline Total (corr.) & 0,00000159546 & 15 & & & \\
\hline
\end{tabular}

$\mathrm{Al}$ analizar cuales factores principales son significativos se observa que la interacción CD presenta significancia con un valor-p cercano a 0.05 pero el factor $\mathrm{C}$ no es significativo y la interacción debe su significancia por el efecto del factor D por ello se decidió descartar el factor C. Para validar los resultados obtenidos del análisis de varianza se evaluaron los supuestos de normalidad, homocedasticidad e independencia, donde se obtuvo que el análisis de varianza cumple con cada uno de ellos.

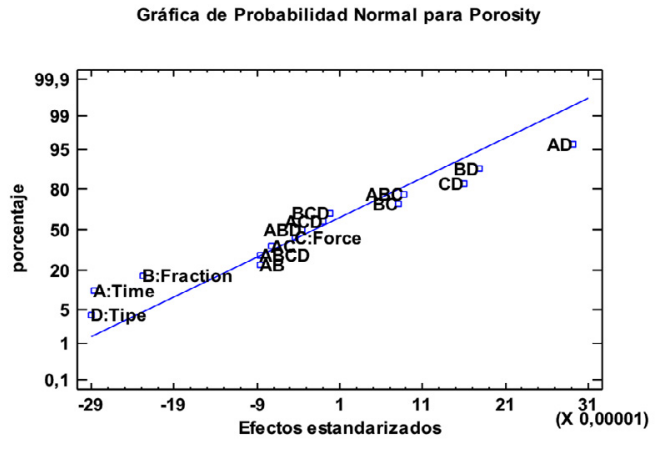

Figura 3. Gráfico de probabilidad normal para los efectos.

Tabla 5. Análisis de varianza (ANOVA) con los factores significativos.

\begin{tabular}{llllll}
\hline Fuente & Suma de Cuadrados & Gl & $\begin{array}{l}\text { Cuadrado } \\
\text { Medio }\end{array}$ & Razón-F & Valor-P \\
\hline A: Tiempo & $3,29247 \mathrm{E}-7$ & 1 & $3,29247 \mathrm{E}-7$ & 13,00 & 0,0048 \\
B: Fracción & $2,08878 \mathrm{E}-7$ & 1 & $2,08878 \mathrm{E}-7$ & 8,25 & 0,0166 \\
D: Tipo & $3,35706 \mathrm{E}-7$ & 1 & $3,35706 \mathrm{E}-7$ & 13,26 & 0,0045 \\
AD & $3,39925 \mathrm{E}-7$ & 1 & $3,39925 \mathrm{E}-7$ & 13,42 & 0,0044 \\
BD & $1,28487 \mathrm{E}-7$ & 1 & $1,28487 \mathrm{E}-7$ & 5,07 & 0,0480 \\
\hline Error total & $2,53218 \mathrm{E}-7$ & 10 & $2,53218 \mathrm{E}-8$ & & \\
\hline Total (corr.) & 0,00000159546 & 15 & & & \\
\hline
\end{tabular}

3.2. Diseño central compuesto.

Luego de comprobar que se cumplieron los supuestos se desarrolló un diseño CCD que utilizó las condiciones experimentales de un diseño factorial $2 \mathrm{k}$, más las condiciones experimentales centrales y los puntos estrella (alfa) rotable y ortogonal para darle mayor libertad al error, como se muestra en la tabla 6 con el cual se determinó el modelo de regresión que describa como los factores inciden en la porosidad del material adsorbente.

Tabla 6. Diseño CCD.

\begin{tabular}{llll}
\hline Condición experimental & Tiempo $(\mathrm{min})$ & Fracción $(\mathrm{gr})$ & Porosidad $(\emptyset)$ \\
\hline 1 & $23,1066(1,41)$ & $10(1,00)$ & 1,0007271 \\
2 & $12,5(0,00)$ & $2,92893(-1,41)$ & 1,0004883 \\
3 & $12,5(0,00)$ & $10(0,00)$ & 1,0006631 \\
4 & $5(-1,00)$ & $5(-1,00)$ & 1,0005488 \\
5 & $12,5(0,00)$ & $17,0711(1,41)$ & 1,0012857 \\
\hline \multicolumn{5}{l}{ Revista Ingenio, 15(1), Enero-Diciembre 2018, pp. 23-30, ISSN 2011-642X - E-ISSN 2389-864X }
\end{tabular}




\begin{tabular}{llll}
6 & $12,5(0,00)$ & $10(0,00)$ & 1,0006352 \\
7 & $20(1,00)$ & $5(-1,00)$ & 1,0007109 \\
8 & $20(1,00)$ & $15(1,00)$ & 1,0010968 \\
9 & $12,5(0,00)$ & $10(0,00)$ & 1,0009579 \\
10 & $12,5(0,00)$ & $10(0,00)$ & 1,0010587 \\
11 & $1,8934(-1,41)$ & $10(0,00)$ & 1,0014719 \\
12 & $12,5(0,00)$ & $10(0,00)$ & 1,0011386 \\
13 & $5(-1,00)$ & $15(1,00)$ & 1,0031087 \\
14 & $12,5(0,00)$ & $10(0,00)$ & 1,0009733 \\
15 & $12,5(0,00)$ & $10(0,00)$ & 1,0018858 \\
16 & $12,5(0,00)$ & $10(0,00)$ & 1,0013715 \\
\hline
\end{tabular}

Ya que en el gráfico de interacciones figura 4, se observó que la porosidad aumenta con el factor D (-1) en su nivel bajo, se seleccionó el carbón activado para desarrollar el CCD para explorar una región cuyo costo de ejecución fuera razonable con los límites de tiempo de desarrollo de la experimentación.
Posteriormente se obtuvieron los datos del análisis de varianza (ANOVA) para la variable de respuesta (Porosidad), donde se determinó que los factores $\mathrm{A}, \mathrm{B}$ y su interacción generan una superficie de respuesta de $2^{\circ}$ orden como se denota en la tabla 7.

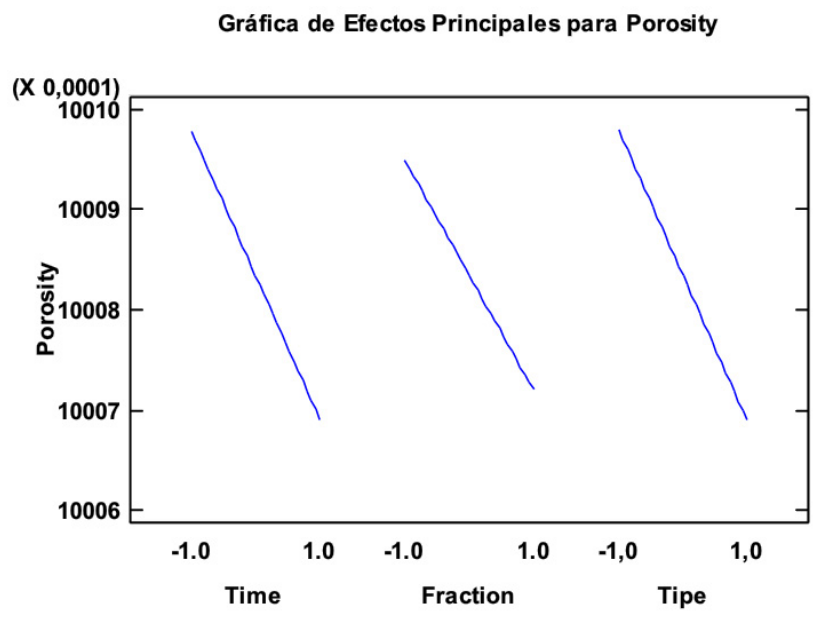

Figura 4. Gráfico de interacciones de los factores.

Tabla 7. Análisis de Varianza (ANOVA) para la porosidad.

\begin{tabular}{llllll}
\hline Fuente & Suma de Cuadrados & Gl & Cuadrado Medio & Razón-F & Valor-P \\
\hline A: Tiempo & 0,00000130194 & 1 & 0,00000130194 & 7,57 & 0,0176 \\
B: Fracción & 0,00000207417 & 1 & 0,00000207417 & 12,05 & 0,0046 \\
AB & $8,5544 \mathrm{E}-7$ & 1 & $8,5544 \mathrm{E}-7$ & 4,97 & 0,0456 \\
\hline Error total & 0,00000206509 & 12 & $1,72091 \mathrm{E}-7$ & & \\
\hline Total (corr.) & 0,00000629664 & 15 & & & \\
\hline
\end{tabular}

3.3. Verificación de los Supuestos para validar los resultados. Buscandovalidar los resultados obtenidos sey la confiabilidad del diseño, se evaluaron los supuestos estadísticos de (Normalidad, Homocedasticidad e Independencia). Para ello se utilizaron los residuos ya que, al cumplirse los supuestos, estos presentan una distribución normal, media cero y varianza constante. 


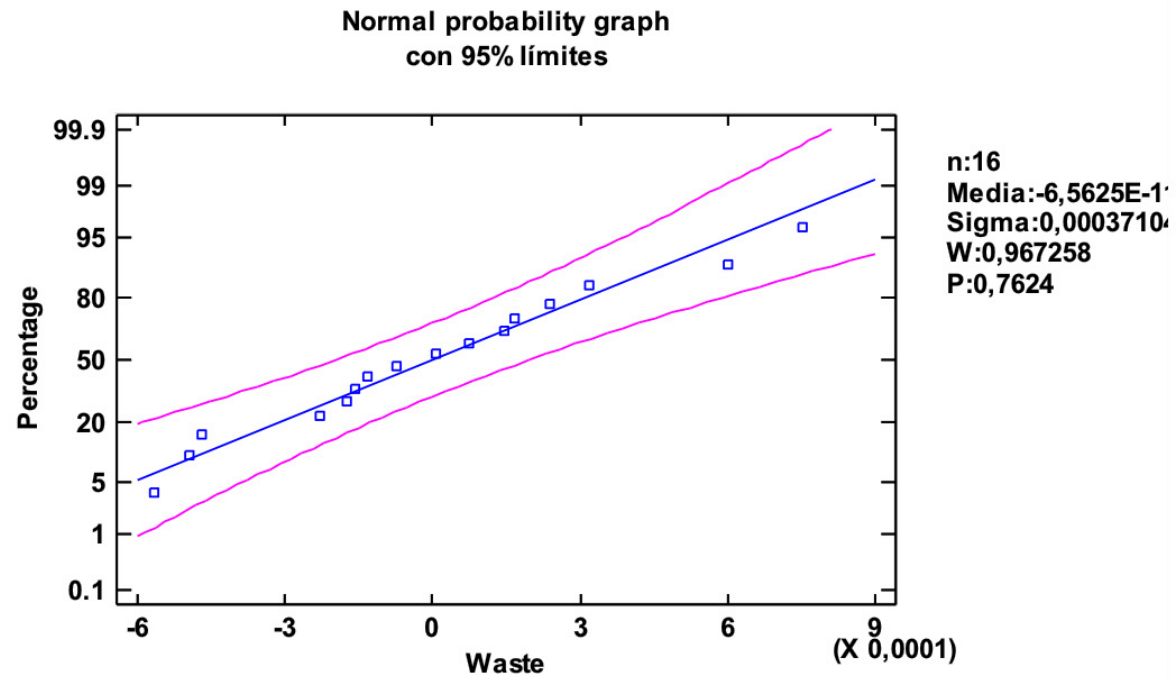

Figura 5. Gráfico de probabilidad normal de los residuales para los residuos de la porosidad.

El primer supuesto en evaluarse fue el de normalidad, donde se observa que los residuos presentan un estado normal para la variable porosidad, como lo representa la figura 5 .
El segundo supuesto evaluado fue la Homocedasticidad, representado a través de la figura 6 , la cual evidencia un comportamiento de la varianza normal. Se observa que las gráficas muestran que los residuales no presentan ningún patrón que indique la no igualdad de varianzas.

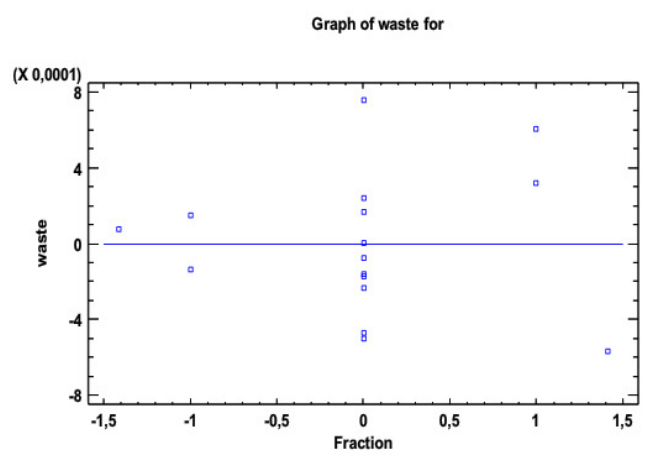

Figura 6. Verificación de igualdad de varianzas.

Por último, se analizó si los residuos presentan independencia como se observa en la figura 7.

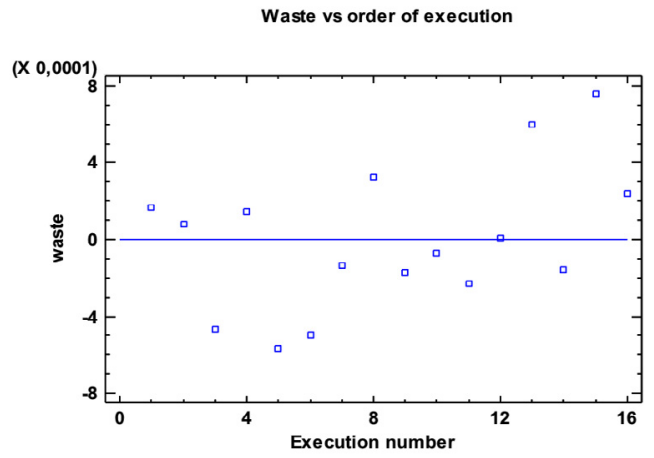

Figura 7. Verificación de igualdad de varianzas.

\subsection{Modelo de Regresión}

Con ayuda de Statgraphics, se obtuvo un modelo de regresión que describe el comportamiento de la variable de respuesta, cuyos coeficientes se muestran en la tabla 8 .

Tabla 8. Coeficientes de regresión.

\begin{tabular}{cc}
\hline Coeficiente & Valor \\
\hline$\beta_{0}$ & 1,00113 \\
$\beta_{1}$ & $-0,000403414$ \\
$\beta_{2}$ & 0,000509188 \\
$\beta_{3}$ & $-0,00046245$ \\
\hline
\end{tabular}

Este modelo descrito en la ecuación 5 se contrastó con los datos experimentales para determinar el error absoluto del modelo y así validarlo como se muestra en la tabla 9. 
Para validar los modelos de regresión se planteó determinar el error absoluto de los datos reales con los datos calculados en el nuevo modelo de regresión estos resultados se observan en la tabla 9.

$$
\emptyset=\beta_{0}-A \beta_{1}+B \beta_{2}-A B \beta_{3}
$$

Tabla 9. Coeficientes de regresión.

\begin{tabular}{cclrrr}
\hline $\begin{array}{l}\text { Condición } \\
\text { experimental }\end{array}$ & Tiempo (min) & Fracción (gr) & Porosidad $(\emptyset)$ & \multicolumn{1}{l}{ Modelo } & \multicolumn{1}{c}{ Error (\%) } \\
\hline 1 & $23,1066(1,41)$ & $10(1,00)$ & 1,0007271 & 1,00055949 & 0,0167490 \\
2 & $12,5(0,00)$ & $2,92893(-1,41)$ & 1,0004883 & 1,00040990 & 0,0078360 \\
3 & $12,5(0,00)$ & $10(0,00)$ & 1,0006631 & 1,00113000 & 0,0466591 \\
4 & $5(-1,00)$ & $5(-1,00)$ & 1,0005488 & 1,00056178 & 0,0149018 \\
5 & $12,5(0,00)$ & $17,0711(1,41)$ & 1,0012857 & 1,00185010 & 0,0563674 \\
6 & $12,5(0,00)$ & $10(0,00)$ & 1,0006352 & 1,00113000 & 0,0494486 \\
7 & $20(1,00)$ & $5(-1,00)$ & 1,0007109 & 1,00067985 & 0,0130976 \\
8 & $20(1,00)$ & $15(1,00)$ & 1,0010968 & 1,00077332 & 0,0323122 \\
9 & $12,5(0,00)$ & $10(0,00)$ & 1,0009579 & 1,00113000 & 0,0171935 \\
10 & $12,5(0,00)$ & $10(0,00)$ & 1,0010587 & 1,00113000 & 0,0071225 \\
11 & $1,8934(-1,41)$ & $10(0,00)$ & 1,0014719 & 1,00170051 & 0,0228276 \\
12 & $12,5(0,00)$ & $10(0,00)$ & 1,0011386 & 1,00113000 & 0,0008590 \\
13 & $5(-1,00)$ & $15(1,00)$ & 1,0031087 & 1,00250505 & 0,0601777 \\
14 & $12,5(0,00)$ & $10(0,00)$ & 1,0009733 & 1,00113000 & 0,0156548 \\
15 & $12,5(0,00)$ & $10(0,00)$ & 1,0018858 & 1,00113000 & 0,0754377 \\
16 & $12,5(0,00)$ & $10(0,00)$ & 1,0013715 & 1,00113000 & 0,0241169 \\
\hline
\end{tabular}

En la figura 8 se muestra la superficie de respuesta para el modelo de regresión generado por Statgraphics. En esta superficie de respuesta se observa que, al aumentar la fracción del material de impregnación y disminuir el tiempo de mezclado la porosidad aumenta, esto debido principalmente a que el cloruro de calcio tiene menor tiempo para ocupar los intersticios del carbón activado.

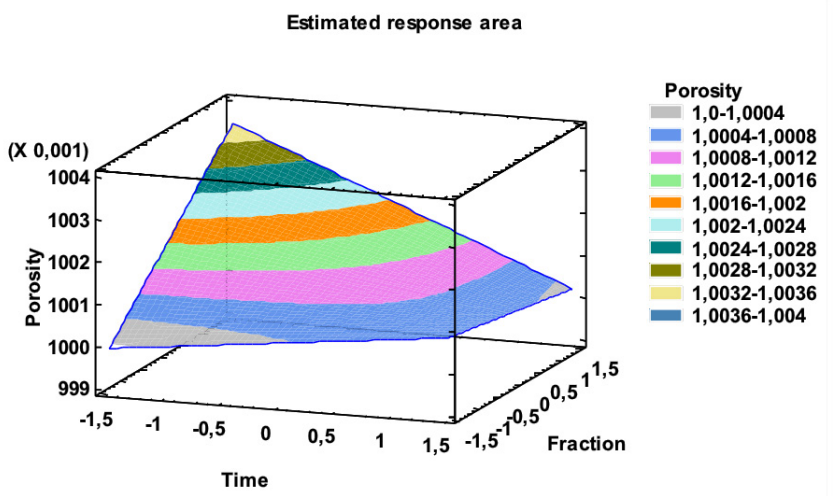

Figura 8. Superficie de respuesta modelo de regresión.

\section{Conclusiones}

Gracias al diseño y las técnicas de experimentación utilizadas en este estudio se determinó cuáles de los efectos individuales e interactivos, son significativos influyen sobre la variable de respuesta porosidad. Se determinó que, el tiempo de homogenización (A), la cantidad de material de impregnación (B)y el tipo de material de impregnación (D), tienen influencia significativa con un $95 \%$ de confiabilidad estadística.

Asimismo, con el diseño factorial $2^{\mathrm{k}}$ se determinó que la fuerza aplicad al material adsorbente compuesto no influye sobre la respuesta de la porosidad. Esto permite disminuir el consumo energético para obtener muestras de material adsorbente de este tipo sin utilizar fuerzas de comparación significativas. También se encontró que el carbón activado es el material de impregnación que mejora la porosidad generando un ahorro económico y energético gracias a su menor costo con referencia al grafito expandido. En la región experimental explorada, la variable de respuesta porosidad tiene un comportamiento óptimo cuando la cantidad de material de impregnación aumenta por encima del 50\%, y el tiempo de homogenización es el mínimo permitido (60s), dado que cuando el tiempo de homogenización es mayor los intersticios libres son ocupados por dicho material.

\section{Referencias Bibliográficas}

[1] E. Pérez, "Diseño y construccion de un sistema de generacion de electricidad a partir del uso de energia solar," Universidad Francisco de Paula Santander Ocaña, 2014.

[2] C. S. Ioan Sarbu, "General review of solar-powered closed sorption refrigeration systems",Energy Conversion and Management, vol. 105, p. 
403-422, 2015. Doi: https://doi.org/10.1016/j. enconman.2015.07.084

[3] Noriega-Sánchez, C. J., \& Pérez-Rojas, E. E. "Sistemas de refrigeración por adsorción como una alternativa para el uso de calor residual y energía solar". Revista Ingenio, 4(1), 7-11, 2012. https://doi. org/10.22463/2011642X.1994

[4] AliAl Alilia, Yunho Hwangb, Reinhard Radermacher. "Review of solar thermal air conditioning technologies". International Journal of Refrigeration, 39 (1), 4-42, 2014. Doi: https://doi.org/10.1016/j. ijrefrig.2013.11.028

[5] Fayazmanesh, K. Salari, S. Bahrami, M. "Effective thermal conductivity modeling of consolidated sorption composites containing graphite flakes." International Journal of Heatand Mass Transfer. 115(1) 73-79, 2017. Doi: https://doi.org/10.1016/j. ijheatmasstransfer.2017.07.020

[6] Cheng, F. Wen, R. Huang, Z. Fang, M. Liu, Y. Wu, $X$. Min, $X$. "Preparation and analysis of lightweight wall material with expanded graphite (EG)/paraffin composites for solar energy storage", Applied Thermal Engineering. 120(6), 107-114, 2017. Doi: https://doi. org/10.1016/j.applthermaleng.2017.03.129

[7] R. W. R. O. T. L. J.K. Kiplagat, "Lithium chloride - Expanded graphite composite sorbent for solar powered ice maker", Solar energy, vol. 84, p. 1587-1594, 2010. Doi: https://doi.org/10.1016/j. solener.2010.06.014

[8] W. R. K. J. W. C. Oliveira RG, "Novel composite sorbent for resorption systems and for chemisorption air conditioners driven by low generation temperature",Renew Energy, vol. 34, p. 2757-2764, 2009. Doi: https://doi.org/10.1016/j. renene.2009.05.016

[9] R. W. G. H. J. Denga, "A review of thermally activated cooling technologies for combined cooling, heating and power systems", Progress in Energy and Combustion Science, vol. 37(2), p. 172-203, 2011. Doi: https://doi.org/10.1016/j.pecs.2010.05.003

[10] W. R. W. C. Oliveira RG, "Evaluation of the cooling performance of a consolidated expanded graphitecalcium chloride reactive bed for chemisorption icemaker", Int J Refrig, vol. 30, p. 103-112, 2007. Doi: https://doi.org/10.1016/j.ijrefrig.2006.08.003

[11] R. W. R. O. T. L. M. L. J.K. Kiplagat, "Experimental study on the effects of the operation conditions on the performance of a chemisorption air conditioner powered by low grade heat", Applied Energy, vol. 1, p. 571-580,2013.Doi: https://doi.org/10.1016/j. apenergy.2012.10.025

[12] H. L. K. Han, «Gas permeability of expanded graphite-metallic salt composite, Appl. Therm.
Eng., vol. 21(4), p. 453-463, 2001.Doi: https://doi. org/10.1016/S1359-4311(00)00056-9 